\title{
有偏抽样下带信息观察和删失的面板数据的 统计分析
}

\author{
刘焕涁 ${ }^{(1)}$ ，苗瑞 ${ }^{(2)}$ ，孙六全 ${ }^{(2 *}$ \\ (1) 黄冈师范学院数学与信息科学学院, 黄冈 438000 ; \\ (2) 中国科学院数学与系统科学研究院, 北京 100190 \\ E-mail: lhb@hgnu.edu.cn, xiaomiaohappy@126.com, slq@amt.ac.cn
}

收稿日期: 2009-12-21；接受日期: 2011-03-14; * 通信作者

国家自然科学基金 (批准号: 10731010, 10971015, 11021161)、国家重点基础研究发展计划 (批准号: 2007CB814902)、中国科学院 随机复杂结构与数据科学重点实验室 (批准号: 2008DP173182) 和湖北省自然科学基金 (批准号: 2008CDB069) 以及湖北省教育厅 自然科学基金重大项目 (批准号: Z20092701) 资助项目

摘要＼cjkstart面板数据经常出现在许多研究领域, 比如纵向跟踪研究. 在很多情况下, 纵向反应变量与观察 时间和删失时间都有关系. 本文在有偏抽样下, 针对这些相关性存在的情况, 利用一个不能观察的潜在 变量, 提出了一个联合建模方法来刻画纵向反应变量与观察时间和删失时间的相关性, 获得了模型中 回归参数的估计方程以及估计的渐近性质, 并通过数值模拟验证了这些估计在小样本下也是有效的, 同时把该估计方法用于一组实际的膀胱癌数据分析中.

关键词 有偏抽样 估计方程 带信息观察过程 联合建模 潜在变量 面板数据

MSC (2000) 主题分类 $62 \mathrm{G} 05,62 \mathrm{~N} 02$

\section{1 引言}

面板数据经常发生在许多研究领域, 比如医学上的跟踪研究和观察调查. 对于这类数据的研究已 经有许多方法 ${ }^{[1-3]}$. 例如, Diggle ${ }^{[1]}$ 总结了一些比较常用的方法, 其中包括估计方程和随机效应模型方 法; Lin 和 Ying ${ }^{[4]}$ 以及 Welsh 等 ${ }^{[5]}$ 提出了一些关于纵向数据的半参数和非参数回归分析方法; Cheng 和 $\mathrm{Wei}^{\left[{ }^{[6]}\right.}$ 建立了一个半参数乘积模型来分析面板数据. 所有上述方法都是基于一个假设: 观察时间和 删失时间对于纵向反应变量是无信息的, 即纵向反应变量与观察时间和删失时间是相互独立的.

一些学者也考虑了带信息删失时间的情况 ${ }^{[7-13]}$. 在这种情况下, 存在一个与纵向反应变量具有 相依性的删失时间 (如死亡). 为了获得有效的统计推断, 必须对纵向变量和删失时间进行联合建模. 在这种情况下提出的各种方法, 一般也是假设观察时间是无信息的.

在许多实际应用中, 纵向反应变量可能既与删失时间有关, 又与观察时间有关. 一个比较常见的 情况就是删失时间和观察时间直接依赖于反应变量. 例如, 在医学研究中, 病人多次住院时间和离院 时间一般与病人本身的病情有关 [14-17]. 另外, 当不规则的抽样时间受到协变量以外的其他因素影响 时, 往往需要对均值反应模型引入一个有偏抽样设计来达到更好的推断效果 $[14,18]$. 目前只有极少的 文献考虑纵向反应变量与观察时间有关的问题 ${ }^{[19-22]}$. 本文的主要目的就是在有偏抽样下, 考虑纵向 反应变量同时与观察时间和删失时间相依的情况. 
本文结构如下: 第 2 节在有偏抽样下对纵向反应变量和观察时间进行联合建模. 在建模时, 我们 用一个半参数随机效应模型来刻画纵向反应变量, 用一个特定个体的非齐次 Poisson 过程来刻画观察 时间过程, 并引入一个潜在变量来描述这三个过程的相依性, 同时删失时间过程可以是任意的. 第 3 节讨论感兴趣的纵向反应变量模型中回归参数的估计, 并研究这些估计的渐近性. 第 4 节讨论模型检 验, 提出一个基于残差的模型评价方法. 第 5 节通过一些数值模拟来验证所提出的方法在小样本下也 是有效的. 第 6 节把所得的方法应用到一组实际的膀胱癌数据分析中. 本文的所有证明在附录中给出.

\section{2 联合模型}

设 $Y(t)$ 为感兴趣的纵向反应变量, $X(t)$ 为 $p \times 1$ 维的可能与时间有关的协变量, $C$ 为删失时间, $N(t)$ 为在时间 $t$ 或之前所经历的观察时间次数的计数过程. 纵向过程 $Y(t)$ 只在计数过程 $N(t)$ 的跳 跃时间点才能被观察到, 其中 $t \leqslant C$. 若 $Y(t), N(t)$ 和 $C$ 在给定协变量下条件独立时, 一般用边际方 法对 $Y(t)$ 进行推断. 这里我们主要是在有偏抽样下考虑它们之间是相依的情况, 也就是说, 观察时间 和删失时间对反应变量是有信息的. 因此, 很自然的通过一个潜在变量来进行联合建模. 下面所提出 的联合模型非常灵活, 不需要对潜在变量和删失时间的分布作任何假设.

设 $V$ 为一个非负的潜在变量. 不失一般性, 我们假设 $Y(t)$ 是非负的 ${ }^{[6]}$, 且在给定 $X(t)$ 和 $V$ 的 条件下, $Y(t)$ 满足边际模型:

$$
E\{Y(t) \mid X(t), V\}=V \mu_{0}(t) \exp \left\{\beta^{\prime} X(t)\right\},
$$

其中 $\mu_{0}(t)$ 为未知非负的连续函数, $\beta$ 为未知回归参数向量. 当上述模型不含有 $V$ 时, 已经被 Cheng 和 $\mathrm{Wei}{ }^{[6]}$ 所研究.

设 $Z$ 为 $q \times 1$ 的协变量, 且 $E(V \mid Z)=1$. 假定在给定 $Z$ 和 $V$ 的情况下, 观察时间过程 $N(t)$ 是一 个非齐次 Poisson 过程, 其强度函数为

$$
\lambda(t \mid Z, V)=V \lambda_{0}(t) \exp \left(\gamma^{\prime} Z\right)
$$

其中 $\gamma$ 为未知的回归参数向量, $\lambda_{0}(t)$ 为未知连续的基本强度函数. 在复发事件数据分析中, 模型 $(2)$ 已经被一些作者使用过 ${ }^{[7,15]}$. 这里我们允许两组协变量 $X(t)$ 和 $Z$ 是任意的. 在没有潜在变量 $V$ 时, 模型 (2) 被称为可调整的预测模型或有偏抽样模型 ${ }^{[14,18]}$. 下面我们假定删失时间 $C$ 以任意方式依赖 于 $X(t), Z$ 和 $V$, 但在 $X(t), Z$ 和 $V$ 给定的条件下, $Y(\cdot), N(\cdot)$ 和 $C$ 相互独立. 因此我们有

$$
E\{d N(t) \mid X(t), Z, V, Y(t), C \geqslant t\}=E\{d N(t) \mid Z, V\}
$$

以及

$$
E\{Y(t) \mid X(t), V, C \geqslant t\}=E\{Y(t) \mid X(t), V\} .
$$

模型 (1) 和 (2) 的一个主要特征是它们允许在有偏抽样下反应过程 $Y(t)$ 和观察时间过程 $N(t)$ 具 有相依性. 在模型 (1) 和 (2) 下, $V$ 的分布、基本函数 $\mu_{0}(t)$ 和 $\lambda_{0}(t)$ 以及 $C$ 的分布都被认为是模型的 非参数部分. 本文的主要兴趣是评价协变量对反应变量的影响, 即估计参数 $\beta$. 为了这个目的, 我们在 下一节将提出一个估计方程的方法. 


\section{3 回归参数估计}

假设有 $n$ 个个体, 且各个个体之间是相互独立的. 对于第 $i$ 个个体, 类似于第 2 节中记号, 相应 地定义 $Y_{i}(t), X_{i}(t), Z_{i}, V_{i}, C_{i}$ 和 $N_{i}(t)$. 另外, 记 $m_{i}$ 为第 $i$ 个个体的总观察次数, $T_{i 1}, \ldots, T_{i, m_{i}}$ 为第 $i$ 个个体的观察时间. 为了调整有偏抽样, 定义

$$
\rho_{i}(t ; \gamma, h)=\frac{\exp \left(\gamma^{\prime} Z_{i}\right)}{h\left(X_{i}(t)\right)},
$$

其中 $h>0$ 为 $X_{i}(t)$ 的一个已知函数. 选择适当的函数 $h$, 对于减少权重的波动性是十分有用的. 在 实际应用中, 正如 Buzkova 和 Lumley ${ }^{[18]}$ 所讨论的那样, 我们可以取 $h_{0}\left(X_{i}(t)\right)=\exp \left\{\theta_{0}^{\prime} X_{i}(t)\right\}$. 关于 $\theta_{0}$ 的选取, 我们可以建立类似于模型 $(2)$ 的比例风险模型, 其中用 $X_{i}(t)$ 代替 $Z_{i}$, 则相应的回归参数 的估计为 $\theta_{0}$ 的最优选择. 当 $Z_{i}$ 是 $X_{i}(t)$ 的一部分时, 则取 $\rho_{i}\left(t ; \gamma, h_{0}\right)=1$.

$V_{i}$ 已知时, 对于给定的 $\gamma$, 利用文献 [6] 和 [18] 的思想, 我们可以用下列方程的解来估计 $\beta$ :

$$
U(\beta ; \gamma)=n^{-1} \sum_{i=1}^{n} \int_{0}^{\tau} Q(t)\left\{X_{i}(t)-\bar{X}^{*}(t ; \beta, h)\right\} \frac{\Delta_{i}(t) Y_{i} d N_{i}(t)}{\rho_{i}(t ; \gamma, h)},
$$

其中 $Q(t)$ 为一个可能依赖于数据的权函数, $\tau$ 为最长的跟踪时间, $\Delta_{i}(t)=I\left(C_{i} \geqslant t\right)$, 以及

$$
\bar{X}^{*}(t ; \beta, h)=\frac{\sum_{i=1}^{n} \Delta_{i}(t) V_{i}^{2} \exp \left\{\beta^{\prime} X_{i}(t)\right\} h\left(X_{i}(t)\right) X_{i}(t)}{\sum_{i=1}^{n} \Delta_{i}(t) V_{i}^{2} \exp \left\{\beta^{\prime} X_{i}(t)\right\} h\left(X_{i}(t)\right)} .
$$

然而在实际中, $V_{i}$ 是不能观察的, 且 $\gamma$ 是未知的, 因此不能直接使用上面的估计方程. 为此, 考虑 模型 $(2)$, 对于 $0 \leqslant t \leqslant \tau$, 定义

$$
\Lambda_{0}(t)=\int_{0}^{t} \lambda_{0}(u) d u, \quad F(t)=\Lambda_{0}(t) / \Lambda_{0}(\tau) .
$$

记 $\left\{s_{(l)}\right\}$ 为观察时间的从小到大的所有不同取值, $d_{(l)}=\sum_{i=1}^{n} d N_{i}\left(s_{(l)}\right)$ 为 $s_{(l)}$ 处的观察数, $N_{(l)}=$ $\sum_{i=1}^{n} \Delta_{i}\left(s_{(l)}\right) N_{i}\left(s_{(l)}\right)$ 为观察时间和删失时间满足 $T_{i j} \leqslant s_{(l)} \leqslant C_{i}$ 时的总观察数. 因此在给定 $\left\{m_{i}, C_{i}\right.$, $\left.V_{i}, Z_{i}\right\}$ 下, 我们得到 $N_{i}(t)$ 的条件似然函数, 从而推导出 $F(t)$ 的非参数极大似然估计为

$$
\hat{F}(t)=\prod_{s_{(l)}>t}\left(1-\frac{d_{(l)}}{N_{(l)}}\right)
$$

(参见文献 [15]). 若没有 $s_{(l)}$ 满足 $s_{(l)}>t$, 则乘积值取为 1 .

记 $\tilde{Z}_{i}=\left(1, Z_{i}^{\prime}\right)^{\prime}, \alpha_{1}=\log \Lambda_{0}(\tau), \alpha=\left(\alpha_{1}, \gamma^{\prime}\right)^{\prime}$. 在模型 (2) 下, 关于 $\alpha$ 的估计, Wang 等 [15] 提出 了下列估计方程:

$$
n^{-1} \sum_{i=1}^{n} W_{i} \tilde{Z}_{i}\left\{m_{i} \hat{F}^{-1}\left(C_{i}\right)-\exp \left(\alpha^{\prime} \tilde{Z}_{i}\right)\right\}=0
$$

其中 $W_{i}$ 为一个可能依赖于 $\left(Z_{i}, \alpha, \hat{F}\right)$ 的权函数. 记 $\hat{\alpha}=\left(\hat{\alpha}_{1}, \hat{\gamma}^{\prime}\right)^{\prime}$ 为上述估计方程 (6) 的解. 注意到

$$
E\left\{m_{i} \mid V_{i}, Z_{i}, C_{i}\right\}=V_{i} \Lambda_{0}\left(C_{i}\right) \exp \left(\gamma^{\prime} Z_{i}\right),
$$

以及

$$
E\left\{m_{i}^{2} \mid V_{i}, Z_{i}, C_{i}\right\}=V_{i}^{2} \Lambda_{0}\left(C_{i}\right)^{2} \exp \left(2 \gamma^{\prime} Z_{i}\right)+V_{i} \Lambda_{0}\left(C_{i}\right) \exp \left(\gamma^{\prime} Z_{i}\right) .
$$


则有

$$
E\left\{\frac{m_{i}\left(m_{i}-1\right)}{\Lambda_{0}\left(C_{i}\right)^{2} \exp \left(2 \gamma^{\prime} Z_{i}\right)} \mid V_{i}, Z_{i}, C_{i}\right\}=V_{i}^{2} .
$$

因此很自然的, $V_{i}^{2}$ 可以用下列 $\hat{\Omega}_{i}$ 来估计:

$$
\hat{\Omega}_{i}=\frac{m_{i}\left(m_{i}-1\right)}{\hat{\Lambda}_{0}\left(C_{i}\right)^{2} \exp \left(2 \hat{\gamma}^{\prime} Z_{i}\right)},
$$

其中 $\hat{\Lambda}_{0}(t)=\hat{F}(t) \exp \left(\hat{\alpha}_{1}\right)$.

在估计方程 (5) 中, 用 $\hat{\Omega}_{i}$ 代替 $V_{i}^{2}$, 我们建议用下列方程的解作为 $\beta$ 的估计:

$$
\hat{U}(\beta ; \gamma)=n^{-1} \sum_{i=1}^{n} \int_{0}^{\tau} Q(t)\left\{X_{i}(t)-\bar{X}(t ; \beta, h)\right\} \frac{\Delta_{i}(t) Y_{i} d N_{i}(t)}{\rho_{i}(t ; \gamma, h)}=0,
$$

其中

$$
\bar{X}(t ; \beta, \gamma)=\frac{\sum_{i=1}^{n} \Delta_{i}(t) \hat{\Omega}_{i} \exp \left\{\beta^{\prime} X_{i}(t)\right\} h\left(X_{i}(t)\right) X_{i}(t)}{\sum_{i=1}^{n} \Delta_{i}(t) \hat{\Omega}_{i} \exp \left\{\beta^{\prime} X_{i}(t)\right\} h\left(X_{i}(t)\right)} .
$$

记方程 (7) 的解为 $\hat{\beta}$. 根据大数定理以及 $\hat{\Lambda}_{0}$ 和 $\hat{\gamma}$ 的相合性, 很容易证明 $\hat{\beta}$ 是相合的 (可参见文献 [6]). 为了研究 $\hat{\beta}$ 的渐近正态性, 定义

$$
\begin{aligned}
\hat{H}(t) & =n^{-1} \sum_{i=1}^{n} \sum_{j=1}^{m_{i}} I\left(T_{i j} \leqslant t\right), \\
\hat{R}(t) & =n^{-1} \sum_{i=1}^{n} \sum_{j=1}^{m_{i}} I\left(T_{i j} \leqslant t \leqslant C_{i}\right), \\
\hat{\mathcal{A}}(t) & =\sum_{i=1}^{n} \int_{0}^{t} \frac{\rho_{i}(t ; \hat{\gamma}, h)^{-1} \Delta_{i}(u) Y_{i}(u) d N_{i}(u)}{\sum_{k=1}^{n} \Delta_{k}(u) \hat{\Omega}_{k} \exp \left\{\hat{\beta}^{\prime} X_{k}(u)\right\} h\left(X_{k}(u)\right)}, \\
\hat{b}_{i}(t) & =\sum_{j=1}^{m_{i}}\left\{\int_{t}^{\tau} \frac{I\left(T_{i j} \leqslant u \leqslant C_{i}\right) d \hat{H}(u)}{\hat{R}^{2}(u)}-\frac{I\left(t<T_{i j} \leqslant \tau\right)}{\hat{R}\left(T_{i j}\right)}\right\}, \\
\hat{e}_{i}(\alpha) & =-\int \frac{w \tilde{z} m \hat{b}_{i}(c) d P_{1 n}(w, \tilde{z}, c, m)}{\hat{F}(c)}+W_{i} \tilde{Z}_{i}\left\{\frac{m_{i}}{\hat{F}\left(C_{i}\right)}-\exp \left(\alpha^{\prime} \tilde{Z}_{i}\right)\right\},
\end{aligned}
$$

其中 $P_{1 n}(w, \tilde{z}, c, m)$ 为 $\left\{\left(W_{i}, \tilde{Z}_{i}, C_{i}, m_{i}\right), i=1, \ldots, n\right\}$ 的经验测度. 令 $\hat{\eta}_{i}(\alpha)$ 为向量函数 $\left[E\left\{-\partial \hat{e}_{i}(\alpha) /\right.\right.$ $\partial \alpha\}]^{-1} \hat{e}_{i}(\alpha)$ 除去第一个分量以外的其它分量, $\hat{\phi}_{i}(\alpha)$ 为 $\left(E\left\{-\partial \hat{e}_{i}(\alpha) / \partial \alpha\right\}\right)^{-1} \hat{e}_{i}(\alpha)$ 的第一个分量, $\hat{\xi}_{i}(t)=$ $\hat{b}_{i}(t)+\hat{\phi}_{i}(\hat{\alpha})$.

记 $\beta_{0}$ 为 $\beta$ 的真值. 在附录中我们证明了在一定正则条件下, $n^{1 / 2}\left(\hat{\beta}-\beta_{0}\right)$ 依分布收敛到一个均值 为零的正态随机向量, 其协方差矩阵的相合估计为 $\hat{D}^{-1} \hat{\Sigma} \hat{D}^{-1}$, 其中 $\hat{\Sigma}=n^{-1} \sum_{i=1}^{n} \hat{\Psi}_{i}^{\otimes 2}$,

$$
\begin{aligned}
\hat{\Psi}_{i}= & \int_{0}^{\tau} Q(t)\left\{X_{i}(t)-\bar{X}(t ; \hat{\beta}, h)\right\} \rho_{i}(t ; \hat{\gamma}, h)^{-1} \Delta_{i}(t) Y_{i}(t) d N_{i}(t) \\
& -\int_{0}^{\tau} Q(t)\left\{X_{i}(t)-\bar{X}(t ; \hat{\beta}, h)\right\} \hat{\Omega}_{i} \exp \left\{\hat{\beta}^{\prime} X_{i}(t)\right\} h\left(X_{i}(t)\right) \Delta_{i}(t) d \hat{\mathcal{A}}(t) \\
& +2 \int_{0}^{\tau} Q(t)\left[\int I(c \geqslant t)\{x(t)-\bar{X}(t ; \hat{\beta}, h)\} h(x(t)) \frac{m(m-1) \exp \left\{\hat{\beta}^{\prime} x(t)\right\}}{\hat{\Lambda}_{0}(c)^{2} \exp \left(2 \hat{\gamma}^{\prime} z\right)}\right. \\
& \left.\times\left\{\hat{\xi}_{i}(c)+z^{\prime} \hat{\eta}_{i}(\hat{\alpha})\right\} d P_{2 n}(z, c, m, x(t))\right] d \hat{\mathcal{A}}(t)-\hat{P} \hat{\eta}_{i}(\hat{\alpha})
\end{aligned}
$$




$$
\hat{P}=n^{-1} \sum_{i=1}^{n} \int_{0}^{\tau} Q(t)\left\{X_{i}(t)-\bar{X}(t ; h)\right\} Z_{i}^{\prime} \rho_{i}(t ; \hat{\gamma}, h)^{-1} \Delta_{i}(t) Y_{i}(t) d N_{i}(t),
$$

以及

$$
\hat{D}=n^{-1} \sum_{i=1}^{n} \int_{0}^{\tau} Q(t)\left\{X_{i}-\bar{X}(t ; \hat{\beta}, h)\right\}^{\otimes 2} \rho_{i}(t ; \hat{\gamma}, h)^{-1} \Delta_{i}(t) Y_{i}(t) d N_{i}(t) .
$$

在上式中, 对于任何向量 $a, a^{\otimes 2}=a a^{\prime}$, 以及对任何 $t \in[0, \tau], P_{2 n}(z, c, m, x(t))$ 代表 $\left\{\left(Z_{i}, C_{i}, m_{i}, X_{i}(t)\right)\right.$, $i=1, \ldots, n\}$ 的经验测度.

\section{4 模型检验}

本节主要研究模型 (1) 的检验问题, 对于模型 (2) 的检验问题, 可以参见文献 [7] 中的一些讨论. 对于 $i=1, \ldots, n$, 定义

$$
\hat{M}_{i}(t)=\int_{0}^{t} \rho_{i}(u ; \hat{\gamma}, h)^{-1}\left[\Delta_{i}(u) Y_{i}(u) d N_{i}(u)-\Delta_{i}(u) \hat{\Omega}_{i} \exp \left\{\hat{\beta}^{\prime} X_{i}(u)+\hat{\gamma}^{\prime} Z_{i}\right\} d \hat{\mathcal{A}}(u)\right] .
$$

类似于文献 [23], 我们考虑如下的累积残差和:

$$
\mathcal{F}(t, y)=n^{-1 / 2} \sum_{i=1}^{n} \int_{0}^{t} I\left(X_{i}(u) \leqslant y\right) d \hat{M}_{i}(u),
$$

其中 $I\left(X_{i}(u) \leqslant y\right)$ 表示对于 $u \in[0, t], X_{i}(u)$ 的各个分量不小于 $y$ 的各个对应分量. 在附录 II 中, 我 们证明了在零假设下, $\mathcal{F}(t ; y)$ 弱收玫到一个零均值的 Gauss 过程, 其在 $(t, y)$ 和 $\left(t^{\dagger}, y^{\dagger}\right)$ 处的协方差函 数的相合估计为 $\hat{\Gamma}\left\{(t, y),\left(t^{\dagger}, y^{\dagger}\right)\right\}$, 其中

$$
\begin{aligned}
& \hat{\Gamma}\left\{(t, y),\left(t^{\dagger}, y^{\dagger}\right)\right\}=n^{-1} \sum_{i=1}^{n} \hat{\Upsilon}_{i}(t, y) \hat{\Upsilon}_{i}\left(t^{\dagger}, y^{\dagger}\right), \\
& \hat{\Upsilon}_{i}(t, y)=\int_{0}^{t}\left\{I\left(X_{i}(u) \leqslant y\right)-\tilde{X}(u, y)\right\} d \hat{M}_{i}(u)+2 \int_{0}^{t}\left[\int\{I(x(u) \leqslant y)-\tilde{X}(u, y)\}\right. \\
&\left.\quad \times h(x(u)) I(c \geqslant u) \frac{m(m-1) \exp \left\{\hat{\beta}^{\prime} x(u)\right\}}{\hat{\Lambda}_{0}(c)^{2} \exp \left(2 \hat{\gamma}^{\prime} z\right)}\left\{\hat{\xi}_{i}(c)+z^{\prime} \hat{\eta}_{i}(\hat{\alpha})\right\} d P_{2 n}(z, c, m, x(u))\right] d \hat{\mathcal{A}}(u) \\
& \quad-B_{x}(t, y) \hat{D}^{-1} \hat{\Psi}_{i}-B_{z}(t, y) \hat{\eta}_{i}(\hat{\alpha}), \\
& B_{x}(t, y)=n^{-1} \sum_{i=1}^{n} \int_{0}^{t}\left\{I\left(X_{i}(u) \leqslant y\right)-\tilde{X}(u, y)\right\} X_{i}(u)^{\prime} \exp \left\{\hat{\beta}^{\prime} X_{i}(u)\right\} h\left(X_{i}(u)\right) \Delta_{i}(u) \hat{\Omega}_{i} d \hat{\mathcal{A}}(u), \\
& B_{z}(t, y)= n^{-1} \sum_{i=1}^{n} \int_{0}^{t}\left\{I\left(X_{i}(u) \leqslant y\right)-\tilde{X}(u, y)\right\} Z_{i}^{\prime} \rho_{i}(u ; \hat{\gamma}, h)^{-1} \Delta_{i}(u) Y_{i}(u) d N_{i}(u),
\end{aligned}
$$

以及

$$
\tilde{X}(t, y)=\frac{\sum_{i=1}^{n} I\left(X_{i}(t) \leqslant y\right) \Delta_{i}(t) \hat{\Omega}_{i} \exp \left\{\hat{\beta}^{\prime} X_{i}(t)\right\} h\left(X_{i}(t)\right)}{\sum_{i=1}^{n} \Delta_{i}(t) \hat{\Omega}_{i} \exp \left\{\hat{\beta}^{\prime} X_{i}(t)\right\} h\left(X_{i}(t)\right)} .
$$


基于上面的结果, 我们很难获得 $\mathcal{F}(t, y)$ 的分布的解析估计. 为此, 我们借助重抽样方法 ${ }^{[23]}$. 记 $\left(G_{1}, \ldots, G_{n}\right)$ 为相互独立的标准正态随机变量而且独立于观察数据. 类似于 [23] 中的证明, 易知 $\mathcal{F}(t, y)$ 在零假设下的分布可由下列零均值的 Gauss 过程逼近:

$$
\hat{\mathcal{F}}(t, y)=n^{-1 / 2} \sum_{i=1}^{n} \hat{\Upsilon}_{i}(t, y) G_{i} .
$$

为了获得 $\mathcal{F}(t, y)$ 的渐近分布, 可以在观察数据给定的情况下, 通过重复产生标准正态随机样本 $\left(G_{1}, \ldots, G_{n}\right)$ 得到大量的 $\hat{\mathcal{F}}(t, y)$ 来实现近似 $\mathcal{F}(t, y)$ 的分布. 为了评价模型 (1) 的整体拟合效果, 可以 画出 $\mathcal{F}(t, y)$ 和许多 $\hat{\mathcal{F}}(t, y)$ 实现的曲线图, 从而直观判断他们是否来自相同总体. 更正式地, 我们可以 利用 Kolmogorov-Smirnov 检验统计量 $\sup _{t, y}|\mathcal{F}(t, y)|$, 其 $p$ 值可以通过比较 $\sup _{t, y}|\mathcal{F}(t, y)|$ 的观察值 与 $\sup _{t, y}|\hat{\mathcal{F}}(t, y)|$ 的大量实现值来得到.

\section{5 数值模拟}

为了验证所提出的估计方法在小样本下的表现, 本节进行一些数值模拟. 由于 Wang 等 ${ }^{[15]}$ 已经 验证了 $\gamma$ 和 $\Lambda_{0}(t)$ 的估计的小样本表现, 这里我们主要集中在 $\beta_{0}$ 的估计上. 在下面的模拟中, 对协变 量 $Z_{i}$, 我们考虑以下两种情况: (i) $Z_{i}$ 为服从正态分布 $N(0,0.25)$ 的随机变量; (ii) $Z_{i}$ 为 Bernoulli 随 机变量, 其成功概率为 0.5 . 当 $Z_{i}$ 来自正态分布 $N(0,0.25)$ 时, 类似于文献 [15], 潜在变量由下式产生: $V_{i}=\exp \left\{-\ln (2.75) I\left(Z_{i} \geqslant 0\right)\right\} V_{i}^{*}$, 其中 $V_{i}^{*}$ 的密度函数为

$$
f\left(v^{*} \mid Z_{i}\right)=I\left(Z_{i} \leqslant 0\right) I\left(0.5 \leqslant v^{*} \leqslant 1.5\right)+\frac{I\left(Z_{i} \geqslant 0\right)}{2.5} I\left(1.5 \leqslant v^{*} \leqslant 4\right) .
$$

当 $Z_{i}$ 来自 Bernoulli 分布时, 我们使用 $V_{i}=\exp \left\{-\ln (2.75) Z_{i}\right\} V_{i}^{*}$, 其中 $V_{i}^{*}$ 的密度函数为

$$
f\left(v^{*} \mid Z_{i}\right)=\left(1-Z_{i}\right) I\left(0.5 \leqslant v^{*} \leqslant 1.5\right)+\frac{Z_{i}}{2.5} I\left(1.5 \leqslant v^{*} \leqslant 4\right) .
$$

很容易验证: $E\left(V_{i} \mid Z_{i}\right)=1$.

对于给定的 $Z_{i}$ 和 $V_{i}$, 观察时间 $T_{i j}$ 由模型 (2) 产生, 其中 $\gamma=1, \lambda_{0}(t)=1$ (齐次 Poisson 过程), 或 $\lambda_{0}(t)=(t+7) / 8$ (非齐次 Poisson 过程). 删失时间 $C_{i}$ 由均匀分布 $U(1,6)$ 产生, 由此可知最长的跟 踪时间为 $\tau=6$.

设 $X_{i}$ 为 Bernoulli 随机变量, 其成功概率为 0.5 . 对于 $X_{i}(t)$, 我们考虑两种情况: 一种是与时间 无关, $X_{i}(t)=X_{i}$; 另一种是与时间有关, $X_{i}(t)=X_{i} t$. 对于纵向变量, 我们假定 $Y_{i}(t)$ 由下式产生:

$$
\log Y_{i}(t)=\log V_{i}+\log \mu_{0}(t)+\beta_{0} X_{i}(t)+\epsilon_{i},
$$

其中 $\epsilon_{i}$ 为服从正态分布 $N(0,0.5)$ 的随机变量. 对于 $\mu_{0}(t)$, 我们也考虑两种情况: 一种是 $\mu_{0}(t)=1+t$; 另一种是 $\mu_{0}(t)=1+2 t^{-1 / 2}$. 对于有偏抽样, 我们选取 $h_{0}\left(X_{i}(t)\right)=\exp \left\{\theta_{0} X_{i}\right\}$, 其中 $\theta_{0}=1$. 对于 $\theta_{0}$ 的其它取值, 模拟结果是类似的. 在我们所有的模拟中, 权函数 $W_{i}=Q(t)=1$, 样本量 $n=100$, 重复 次数为 1000 次.

表 1 给出了当观察时间为齐次 Poisson 过程, $\beta_{0}=-0.5,0,0.5$ 时的模拟结果. 表中包含有估计的 Bias, SE, SSE 和 CP, 其中 Bias 为估计 $\hat{\beta}$ 的样本均值减去真值, SE 为 $\hat{\beta}$ 的标准差估计的平均值, SSE 为 $\hat{\beta}$ 的样本标准差, $\mathrm{CP}$ 为 $\beta_{0}$ 的 $95 \%$ 经验覆盖率. 从这些结果可以看出, 我们提出的估计是渐近无偏 的, 其方差估计和覆盖概率是合理的. 
表 1 观察时间为齐次 Poisson 过程的模拟结果

\begin{tabular}{|c|c|c|c|c|c|c|c|c|c|}
\hline \multirow[b]{2}{*}{$\mu_{0}(t)$} & \multirow[b]{2}{*}{$\beta_{0}$} & \multicolumn{4}{|c|}{$Z$ 为正态分布 } & \multicolumn{4}{|c|}{$Z$ 为 Bernoulli 分布 } \\
\hline & & Bias & $\mathrm{SE}$ & SEE & $\mathrm{CP}$ & Bias & SE & SEE & $\mathrm{CP}$ \\
\hline \multicolumn{10}{|c|}{$X(t)$ 与时间无关 } \\
\hline \multirow[t]{3}{*}{$1+t$} & -0.5 & 0.0007 & 0.1575 & 0.1441 & 0.9260 & -0.0069 & 0.1361 & 0.1271 & 0.9290 \\
\hline & 0.0 & 0.0020 & 0.1574 & 0.1451 & 0.9270 & 0.0075 & 0.1481 & 0.1278 & 0.9220 \\
\hline & 0.5 & 0.0051 & 0.1610 & 0.1453 & 0.9220 & 0.0065 & 0.1340 & 0.1278 & 0.9390 \\
\hline \multirow[t]{3}{*}{$1+2 t^{-1 / 2}$} & -0.5 & -0.0060 & 0.1933 & 0.1688 & 0.9350 & 0.0065 & 0.1575 & 0.1441 & 0.9260 \\
\hline & 0.0 & 0.0026 & 0.1953 & 0.1698 & 0.9280 & 0.0052 & 0.1712 & 0.1510 & 0.9360 \\
\hline & 0.5 & 0.0102 & 0.1902 & 0.1715 & 0.9330 & 0.0079 & 0.1760 & 0.1525 & 0.9510 \\
\hline \multicolumn{10}{|c|}{$X(t)$ 与时间有关 } \\
\hline \multirow[t]{3}{*}{$1+t$} & -0.5 & 0.0032 & 0.0623 & 0.0536 & 0.8960 & 0.0015 & 0.0542 & 0.0481 & 0.9110 \\
\hline & 0.0 & 0.0021 & 0.0610 & 0.0540 & 0.9130 & 0.0030 & 0.0523 & 0.0477 & 0.9100 \\
\hline & 0.5 & 0.0032 & 0.0601 & 0.0540 & 0.9180 & 0.0073 & 0.0516 & 0.0488 & 0.9243 \\
\hline \multirow[t]{3}{*}{$1+2 t^{-1 / 2}$} & -0.5 & 0.0031 & 0.0600 & 0.0535 & 0.9270 & 0.0027 & 0.0517 & 0.0470 & 0.9150 \\
\hline & 0.0 & 0.0035 & 0.0576 & 0.0539 & 0.9200 & 0.0015 & 0.0499 & 0.0478 & 0.9350 \\
\hline & 0.5 & 0.0061 & 0.0588 & 0.0543 & 0.9180 & 0.0003 & 0.0512 & 0.0478 & 0.9240 \\
\hline
\end{tabular}

表 2 观察时间为非齐次 Poisson 过程的模拟结果

\begin{tabular}{|c|c|c|c|c|c|c|c|c|c|}
\hline \multirow[b]{2}{*}{$\mu_{0}(t)$} & \multirow[b]{2}{*}{$\beta_{0}$} & \multicolumn{4}{|c|}{$Z$ 为正态分布 } & \multicolumn{4}{|c|}{$Z$ 为 Bernoulli 分布 } \\
\hline & & Bias & $\mathrm{SE}$ & SEE & $\mathrm{CP}$ & Bias & $\mathrm{SE}$ & SEE & $\mathrm{CP}$ \\
\hline \multicolumn{10}{|c|}{$X(t)$ 与时间无关 } \\
\hline \multirow[t]{3}{*}{$1+t$} & -0.5 & 0.0061 & 0.1464 & 0.1342 & 0.9240 & 0.0055 & 0.1379 & 0.1274 & 0.9290 \\
\hline & 0.0 & -0.0064 & 0.1472 & 0.1339 & 0.9200 & 0.0053 & 0.1364 & 0.1276 & 0.9310 \\
\hline & 0.5 & 0.0007 & 0.1492 & 0.1358 & 0.9230 & -0.0028 & 0.1473 & 0.1284 & 0.9040 \\
\hline \multirow[t]{3}{*}{$1+2 t^{-1 / 2}$} & -0.5 & -0.0019 & 0.1789 & 0.1555 & 0.9270 & 0.0074 & 0.1575 & 0.1380 & 0.9300 \\
\hline & 0.0 & 0.0019 & 0.1866 & 0.1563 & 0.9280 & 0.0030 & 0.1712 & 0.1401 & 0.9280 \\
\hline & 0.5 & 0.0035 & 0.1737 & 0.1579 & 0.9100 & -0.0020 & 0.1760 & 0.1385 & 0.9320 \\
\hline \multicolumn{10}{|c|}{$X(t)$ 与时间有关 } \\
\hline \multirow[t]{3}{*}{$1+t$} & -0.5 & 0.0013 & 0.0518 & 0.0464 & 0.9290 & 0.0020 & 0.0454 & 0.0413 & 0.9240 \\
\hline & 0.0 & 0.0018 & 0.0537 & 0.0469 & 0.9270 & -0.0008 & 0.0464 & 0.0414 & 0.9230 \\
\hline & 0.5 & 0.0007 & 0.0505 & 0.0464 & 0.9220 & 0.0000 & 0.0476 & 0.0409 & 0.9010 \\
\hline \multirow[t]{3}{*}{$1+2 t^{-1 / 2}$} & -0.5 & 0.0003 & 0.0488 & 0.0458 & 0.9250 & 0.0011 & 0.0454 & 0.0407 & 0.9170 \\
\hline & 0.0 & 0.0041 & 0.0507 & 0.0464 & 0.9240 & 0.0044 & 0.0448 & 0.0415 & 0.9260 \\
\hline & 0.5 & 0.0052 & 0.0516 & 0.0470 & 0.9170 & 0.0015 & 0.0457 & 0416 & 0.9160 \\
\hline
\end{tabular}

表 2 给出了观察时间为非齐次 Poisson 过程, 其他参数设置与表 1 一样的模拟结果. 其结果也显 示我们所提出的估计方法表现很好. 我们同时也考虑了参数的其它设置方式, 包括增大每个个体的平 均观察次数, 增大样本量以及增大删失时间 $C_{i}$ 的取值范围, 所得到的结果都是类似的. 


\section{6 应用}

本节把所提出的估计方法应用到一组纵向膀胱癌数据分析中 $[21,22]$. 该数据包括 85 个膀胱癌病 人, 其中安慰剂组 47 人, 噻替派治疗组 38 人. 对每个病人, 可观察的信息包括入院诊断时间或观察时 间 (按月) 以及每两次诊断之间新生长的肿瘤个数. 另外, 两个基本的协变量也被记录: 一个是病人进 入研究之前的初始肿瘤个数; 一个是最大的初始肿瘤尺寸. 对所有病人而言, 最长的观察时间是 53 个 月. 该研究的主要目的之一是评价噻替派对膀胱癌肿瘤复发的治疗效果. 我们这里的主要目的是在允 许肿瘤复发与入院诊断时间可能相关的情况下来估计治疗和协变量的效果.

对于 $i=1, \ldots, 85$, 定义 $Y_{i}(t)$ 为在时间 $t$ 处观察到的肿瘤数目. 若病人在安慰剂组, 则定义 $X_{i 1}=0$, 否则为 1 . 记 $X_{i 2}$ 为病人的初始肿瘤个数, $X_{i 3}$ 为病人的最大的初始肿瘤尺寸. 由于最大的 初始肿瘤尺寸已经被证明对观察时间没有影响 ${ }^{[16,17]}$, 因此我们只考虑噻替派治疗和初始肿瘤个数对 观察时间的影响, 并令 $Z_{i 1}=X_{i 1}, Z_{i 2}=X_{i 2}$. 由于 $Z_{i}$ 与 $X_{i}(t)$ 是相同的, 根据文献 [18], 我们选择 $\rho\left(t ; \gamma, h_{0}\right)=1$. 对于 $\theta_{0}$ 的其它选择, 结果是类似的.

应用我们的方法, 并取权函数 $W_{i}(t)=Q(t)=1$ 得到 $\hat{\beta}_{1}=-1.6709, \hat{\beta}_{2}=0.2182, \hat{\beta}_{3}=-0.0901$, 它 们的标准差估计分别为 $0.6114,0.1268,0.1405$. 同时, 我们也得到 $\hat{\gamma_{1}}=0.4786, \hat{\gamma_{2}}=-0.0378$, 其标准 差估计分别为 0.2358 和 0.0516. 这些结果显示, 肿瘤复发过程和观察过程是相关的, 同时噻替派治疗 对肿瘤的复发率有显著影响, 即噻替派治疗显著降低了肿瘤的复发率, 而且病人的初始肿瘤个数越多, 肿瘤的复发率越高. 所获得的结果与 Sun 等 ${ }^{[21,22]}$ 和 Liang 等 ${ }^{[24]}$ 的结果是类似.

最后, 我们用第 4 节的模型检验方法来评估模型 (1) 对这组数据的拟合效果. 通过 $\hat{\mathcal{F}}(t, y)$ 的 1000 次实现, 我们得到 $\sup _{t, y}|\mathcal{F}(t, y)|$ 的 $p$ 值为 0.3980 . 这个结果表明, 模型 (1) 能很好的拟合这组膀脱癌 数据.

致谢 作者衰心感谢编委和审稿专家对本文提出的宝贵意见.

\section{参考文献}

1 Diggle P J, Liang K Y, Zeger S L. The Analysis of Longitudinal Data. Oxford: Oxford University Press, 1994

2 Laird N M, Ware J H. Random-effects models for longitudinal data. Biometrics, 1982, 38: 963-974

3 Zeger S L, Diggle P J. Semiparametric models for longitudinal data with application to CD4 cell numbers in HIV seroconverters. Biometrics, 1994, 50: 689-699

4 Lin D Y, Ying Z. Semiparametric and nonparametric regression analysis of longitudinal data. J Amer Statist Assoc, 2001, 96: 103-126

5 Welsh A H, Lin X, Carroll R J. Marginal longitudinal nonparametric regression: locality and efficiency of spline and kernel methods. J Amer Statist Assoc, 2002, 97: 482-493

6 Cheng S C, Wei L J. Inference for a semiparametric model with panel data. Biometrika, 2000, 87: 89-97

7 Huang C Y, Wang M C. Joint modeling and estimation for recurrent event processes and failure time data. J Amer Statist Assoc, 2004, 99: 1153-1165

8 Little R J A. Modeling the drop-out mechanism in repeated-measures studies. J Amer Statist Assoc, 1995, 90: 11121121

9 Roy J, Lin X. Analysis of multivariate longitudinal outcomes with nonignorable dropouts and missing covariates: changes in methadone treatment practices. J Amer Statist Assoc, 2002, 97: 40-52

10 Sun J, Song X K. Statistical analysis of repeated measurements with informative censoring times. Stat Med, 2001, 20: $63-73$

11 Wang Y, Taylor M G. Jointly modeling longitudinal and event time data with application to acquired immunodeficiency syndrome. J Amer statist Assoc, 2001, 96: 895-905

12 Wu M C, Carroll R J. Estimation and comparison of changes in the presence of informative right censoring by modeling 
the censoring process. Biometrics, 1988, 44: 175-188

13 Wulfsohn M S, Tsiatis A A. A joint model for survival and longitudinal data measured with error. Biometrics, 1997, 53: $330-339$

14 Lin H, Scharfstein D O, Rosenheck D O. Analysis of longitudinal data with irregular outcome-dependent follow-up. J Roy Statist Soc Ser B, 2004, 66: 791-813

15 Wang M C, Qin J, Chiang C T. Analyzing recurrent event data with informative censoring. J Amer Statist Assoc, 2001, 96: 1057-1065

16 Sun J, Wei L J. Regression analysis of panel count data with covariate-dependent observation and censoring times. J Roy Statist Soc Ser B, 2000, 62: 293-302

17 Zhang Y. A semiparametric pseudolikelihood estimation method for panel count data. Biometrika, 2002, 89: 39-48

18 Buzkova P, Lumley T. Semiparametric log-linear regression for longitudinal measurements subject to outcomedependent follow-up. J Statist Plann Inference, 2008, 138: 2450-2461

19 Lipsitz S R, Fitzmaurice G M, Ibrahim J G, et al. Parameter estimation in longitudinal studies with outcome-dependent follow-up. Biometrics, 2002, 58: 621-630

20 Robins J M, Rotnitzky A, Zhao L P. Analysis of semiparametric regression models for repeated outcomes in the presence of missing data. J Amer Statist Assoc, 1995, 90: 106-121

21 Sun J, Park D H, Sun L, et al. Semiparametric regression analysis of longitudinal data with informative observation times. J Amer Statist Assoc, 2005, 100: 882-889

22 Sun J, Sun L, Liu D. Regression analysis of longitudinal data in the presence of informative observation and censoring times. J Amer Statist Assoc, 2007, 102: 1397-1406

23 Lin D Y, Wei L J, Yang I, et al. Semiparametric regression for the mean and rate functions of recurrent events. J Roy Statist Soc Ser B, 2000, 62: 711-730

24 Liang Y, Lu W, Ying Z. Joint modeling and analysis of longitudinal data with informative observation times. Biometrics, 2009, 65: 377-384

\section{附录 I: $\hat{\boldsymbol{\beta}}$ 的渐近正态性}

在这部分中, 我们使用和前面相同的记号, 而且所有极限都是在 $n \rightarrow \infty$ 下得到. 定义

$$
\begin{aligned}
& S^{(0)}(t ; \beta, h)=n^{-1} \sum_{i=1}^{n} \Delta_{i}(t) \hat{\Omega}_{i} \exp \left\{\beta^{\prime} X_{i}(t)\right\} h\left(X_{i}(t)\right), \\
& S^{(1)}(t ; \beta, h)=n^{-1} \sum_{i=1}^{n} \Delta_{i}(t) \hat{\Omega}_{i} \exp \left\{\beta^{\prime} X_{i}(t)\right\} h\left(X_{i}(t)\right) X_{i}(t) .
\end{aligned}
$$

另外 $s^{(0)}(t ; h)$ 和 $s^{(1)}(t ; h)$ 分别是 $S^{(0)}\left(t ; \beta_{0}, h\right)$ 和 $S^{(1)}\left(t ; \beta_{0}, h\right)$ 的极限. 为了推导 $\hat{\beta}$ 的渐近分布, 我们 需要下列正则条件:

(R1) $P(C \geqslant \tau, V>0)>0, E\left(V^{2}\right)<\infty$;

(R2) $X(t), Z$ 和 $h(X(t))$ 都在 $[0, \tau]$ 上有界, 且 $G(t)=E\left\{V \exp \left(\gamma_{0}^{\prime} Z\right) I(C \geqslant t)\right\}$ 为 $t \in[0, \tau]$ 的连 续函数, 其中 $\gamma_{0}$ 是 $\gamma$ 的真值

(R3) 权函数 $Q(t)$ 为一个有界变差函数, 且关于 $t \in[0, \tau]$ 依概率一致收玫于一个确定性函数 $q(t)$;

(R4) $D$ 为非奇异矩阵, 其中 $D=E\left[\int_{0}^{\tau} q(t)\left\{X_{i}(t)-\bar{x}(t ; h)\right\}^{\otimes 2} \rho_{i}\left(t ; \gamma_{0}, h\right)^{-1} \Delta_{i}(t) Y_{i}(t) d N_{i}(t)\right]$, 以及 $\bar{x}(t ; h)=s^{(1)}(t ; h) / s^{(0)}(t ; h)$.

$$
\begin{aligned}
\text { 记 } R(t)=G(t) \Lambda_{0}(t), H(t)=\int_{0}^{t} G(u) d \Lambda_{0}(u), \\
b_{i}(t)=\sum_{j=1}^{m_{i}}\left\{\int_{t}^{\tau} \frac{I\left(T_{i j} \leqslant u \leqslant C_{i}\right) d H(u)}{R^{2}(u)}-\frac{I\left(t<T_{i j} \leqslant \tau\right)}{R\left(T_{i j}\right)}\right\},
\end{aligned}
$$


以及

$$
e_{i}(\alpha)=-\int \frac{w \tilde{z} m b_{i}(c) d P_{1}(w, \tilde{z}, c, m)}{F(c)}+W_{i} \tilde{Z}_{i}\left\{\frac{m_{i}}{F\left(C_{i}\right)}-\exp \left(\alpha^{\prime} \tilde{Z}_{i}\right)\right\}
$$

其中 $P_{1}(w, \tilde{z}, c, m)$ 是 $\left(W_{i}, \tilde{Z}_{i}, C_{i}, m_{i}\right)$ 的联合概率测度. 定义 $\eta_{i}(\alpha)$ 为向量函数 $\left(-\partial e_{i}(\alpha) / \partial \alpha\right)^{-1} e_{i}(\alpha)$ 除去第一个分量以外的其他分量, $\phi_{i}(\alpha)$ 为 $\left(-\partial e_{i}(\alpha) / \partial \alpha\right)^{-1} e_{i}(\alpha)$ 的第一个分量, 以及 $\xi_{i}(t ; \alpha)=b_{i}(t)+$ $\phi_{i}(\alpha)$. 在条件 $(\mathrm{R} 1)$ 和 $(\mathrm{R} 2)$ 下, 由 $[15]$ 知

$$
\begin{aligned}
& n^{1 / 2}\left(\hat{\Lambda}_{0}(t)-\Lambda_{0}(t)\right)=n^{-1 / 2} \Lambda_{0}(t) \sum_{i=1}^{n} \xi_{i}\left(t ; \alpha_{0}\right)+o_{p}(1), \\
& n^{1 / 2}\left(\hat{\gamma}-\gamma_{0}\right)=n^{-1 / 2} \sum_{i=1}^{n} \eta_{i}\left(\alpha_{0}\right)+o_{p}(1)
\end{aligned}
$$

其中 $\alpha_{0}$ 是 $\alpha$ 的真值. 记 $\tilde{\Omega}_{i}=m_{i}\left(m_{i}-1\right) /\left(\Lambda_{0}\left(C_{i}\right)^{2} \exp \left(2 \gamma_{0}^{\prime} Z_{i}\right)\right), \mathcal{A}(t)=\int_{0}^{t} \mu_{0}(u) d \Lambda_{0}(u)$. 由 (A.1), (A.2), Taylor 展开以及控制收敛定理有

$$
\begin{aligned}
n^{1 / 2} \int_{0}^{\tau} q(t) S^{(1)}\left(t ; \beta_{0}, h\right) d \mathcal{A}(t)= & n^{-1 / 2} \sum_{i=1}^{n} \int_{0}^{\tau} q(t) \tilde{\Omega}_{i} \exp \left\{\beta_{0}^{\prime} X_{i}(t)\right\} h\left(X_{i}(t)\right) X_{i}(t) \Delta_{i}(t) d \mathcal{A}(t) \\
& +n^{-1 / 2} \sum_{i=1}^{n} \int_{0}^{\tau} q(t)\left\{\hat{\Omega}_{i}-\tilde{\Omega}_{i}\right\} \exp \left\{\beta_{0}^{\prime} X_{i}(t)\right\} h\left(X_{i}(t)\right) X_{i}(t) \Delta_{i}(t) d \mathcal{A}(t) \\
= & n^{-1 / 2} \sum_{i=1}^{n} \int_{0}^{\tau} q(t) \tilde{\Omega}_{i} \exp \left\{\beta_{0}^{\prime} X_{i}(t)\right\} h\left(X_{i}(t)\right) X_{i}(t) \Delta_{i}(t) d \mathcal{A}(t) \\
& -2 n^{-1 / 2} \sum_{i=1}^{n} \int_{0}^{\tau} q(t)\left[\int I(c \geqslant t) x(t) \frac{m(m-1) \exp \left\{\beta_{0}^{\prime} x(t)\right\} h(x(t))}{\Lambda_{0}(c)^{2} \exp \left(2 \gamma_{0}^{\prime} z\right)}\right. \\
& \left.\times\left\{\xi_{i}\left(c ; \alpha_{0}\right)+z^{\prime} \eta_{i}\left(\alpha_{0}\right)\right\} d P_{2}(z, c, m, x(t))\right] d \mathcal{A}(t)+o_{p}(1),
\end{aligned}
$$

其中对任何 $t \in[0, \tau], P_{2}(z, c, m, x(t))$ 是 $\left(Z_{i}, C_{i}, m_{i}, X_{i}(t)\right)$ 的联合概率测度. 类似地,

$$
\begin{aligned}
n^{1 / 2} & \int_{0}^{\tau} q(t) \bar{x}(t ; h) S^{(0)}\left(t ; \beta_{0}, h\right) d \mathcal{A}(t) \\
= & n^{-1 / 2} \sum_{i=1}^{n} \int_{0}^{\tau} q(t) \bar{x}(t ; h) \tilde{\Omega}_{i} \exp \left\{\beta_{0}^{\prime} X_{i}(t)\right\} h\left(X_{i}(t)\right) \Delta_{i}(t) d \mathcal{A}(t) \\
& -2 n^{-1 / 2} \sum_{i=1}^{n} \int_{0}^{\tau} q(t) \bar{x}(t ; h)\left[\int I(c \geqslant t) \frac{m(m-1) \exp \left\{\beta_{0}^{\prime} x(t)\right\} h(x(t))}{\Lambda_{0}(c)^{2} \exp \left(2 \gamma_{0}^{\prime} z\right)}\right. \\
& \left.\times\left\{\xi_{i}\left(c ; \alpha_{0}\right)+z^{\prime} \eta_{i}\left(\alpha_{0}\right)\right\} d P_{2}(z, c, m, x(t))\right] d \mathcal{A}(t)+o_{p}(1) .
\end{aligned}
$$

注意到估计方程 $\hat{U}(\beta ; \gamma)$ 可以写为

$$
\begin{aligned}
\hat{U}(\beta ; \gamma)= & n^{-1} \sum_{i=1}^{n} \int_{0}^{\tau} Q(t) X_{i}(t) \rho_{i}(t ; \gamma, h)^{-1} \Delta_{i}(t) Y_{i}(t) d N_{i}(t) \\
& -n^{-1} \sum_{i=1}^{n} \int_{0}^{\tau} Q(t) \frac{S^{(1)}(t ; \beta, h)}{S^{(0)}(t ; \beta, h)} \rho_{i}(t ; \gamma, h)^{-1} \Delta_{i}(t) Y_{i}(t) d N_{i}(t),
\end{aligned}
$$


并由大数定理知, $\hat{U}\left(\beta_{0} ; \gamma_{0}\right) \rightarrow 0$. 因此, 根据 (A.3), (A.4) 和文献 [4, 引理 A.1] 以及函数型 Taylor 展 开, 我们有

$$
\begin{aligned}
n^{1 / 2} \hat{U}\left(\beta_{0} ; \gamma_{0}\right)= & n^{-1 / 2} \sum_{i=1}^{n} \int_{0}^{\tau} q(t) X_{i}(t) \rho_{i}\left(t ; \gamma_{0}, h\right)^{-1} \Delta_{i}(t) Y_{i}(t) d N_{i}(t) \\
& -n^{1 / 2} \int_{0}^{\tau} q(t) S^{(1)}\left(t ; \beta_{0}, h\right) d \mathcal{A}(t)+n^{1 / 2} \int_{0}^{\tau} q(t) \bar{x}(t ; h) S^{(0)}\left(t ; \beta_{0}, h\right) d \mathcal{A}(t) \\
& -n^{-1 / 2} \sum_{i=1}^{n} \int_{0}^{\tau} q(t) \bar{x}(t ; h) \rho_{i}\left(t ; \gamma_{0}, h\right)^{-1} \Delta_{i}(t) Y_{i}(t) d N_{i}(t)+o_{p}(1) \\
= & n^{-1 / 2} \sum_{i=1}^{n} \Phi_{i}+o_{p}(1) .
\end{aligned}
$$

由此可见 $n^{1 / 2} \hat{U}\left(\beta_{0} ; \gamma_{0}\right)$ 可以表示为 $n$ 个独立的零均值随机向量之和, 加上一个渐近可忽略的项, 其中

$$
\begin{aligned}
\Phi_{i}= & \int_{0}^{\tau} q(t)\left\{X_{i}(t)-\bar{x}(t ; h)\right\} \rho_{i}\left(t ; \gamma_{0}, h\right)^{-1} \Delta_{i}(t) Y_{i}(t) d N_{i}(t) \\
& -\int_{0}^{\tau} q(t)\left\{X_{i}(t)-\bar{x}(t ; h)\right\} \tilde{\Omega}_{i} \exp \left\{\beta_{0}^{\prime} X_{i}(t)\right\} h\left(X_{i}(t)\right) \Delta_{i}(t) d \mathcal{A}(t) \\
& +2 \int_{0}^{\tau} q(t)\left[\int I(c \geqslant t)\{x(t)-\bar{x}(t ; h)\} \frac{m(m-1) \exp \left\{\beta_{0}^{\prime} x(t)\right\} h(x(t))}{\Lambda_{0}(c)^{2} \exp \left(2 \gamma_{0}^{\prime} z\right)}\right. \\
& \left.\times\left\{\xi_{i}\left(c ; \alpha_{0}\right)+z^{\prime} \eta_{i}\left(\alpha_{0}\right)\right\} d P_{2}(z, c, m, x(t))\right] d \mathcal{A}(t) .
\end{aligned}
$$

易证 $-\partial \hat{U}\left(\beta_{0} ; \gamma\right) /\left.\partial \gamma\right|_{\gamma=\gamma_{0}}$ 依概率收敛到

$$
P=E\left[\int_{0}^{t} q(t)\left\{X_{i}(t)-\bar{x}(t ; h)\right\} Z_{i}^{\prime} \rho_{i}\left(t ; \gamma_{0}, h\right)^{-1} \Delta_{i}(t) Y_{i}(t) d N_{i}(t)\right] .
$$

由 $\hat{U}\left(\beta_{0} ; \hat{\gamma}\right)$ 在 $\hat{U}\left(\beta_{0} ; \gamma_{0}\right)$ 处的 Taylor 展开, 并结合 $\hat{\gamma}$ 的相合性和 (A2), 我们可以得到

$$
n^{1 / 2} \hat{U}\left(\beta_{0} ; \hat{\gamma}\right)=n^{1 / 2} \hat{U}\left(\beta_{0} ; \gamma_{0}\right)-P n^{-1 / 2} \sum_{i=1}^{n} \eta_{i}\left(\alpha_{0}\right)+o_{p}(1) .
$$

于是由多元中心极限定理以及 (A.5) 和 (A.6) 知, $n^{1 / 2} \hat{U}\left(\beta_{0} ; \hat{\gamma}\right)$ 依分布收敛到一个均值为零且协方差矩 阵为 $\Sigma=E\left\{\Psi_{i}^{\otimes 2}\right\}$ 的正态随机向量, 其中 $\Psi_{i}=\Phi_{i}-P \eta_{i}\left(\alpha_{0}\right)$.

注意到 $-\partial \hat{U}\left(\beta_{0} ; \hat{\gamma}\right) / \partial \beta$ 依概率收敛于 $D($ 见条件 $(\mathrm{R} 4))$. 则由 $\hat{U}(\hat{\beta} ; \hat{\gamma})$ 在 $\hat{U}\left(\beta_{0} ; \hat{\gamma}\right)$ 处的 Taylor 展 开, 可得

$$
n^{1 / 2}\left(\hat{\beta}-\beta_{0}\right)=D^{-1} n^{1 / 2} \hat{U}\left(\beta_{0} ; \hat{\gamma}\right)+o_{p}(1) .
$$

因此由 (A.6) 和 (A.7) 知, $n^{1 / 2}\left(\hat{\beta}-\beta_{0}\right)$ 渐近于一个均值为零的正态随机向量, 其协方差矩阵为 $D^{-1} \Sigma D^{-1}$. 利用文献 [23] 中附录 A.3 的证明方法可知, $D^{-1} \Sigma D^{-1}$ 的一个相合估计为 $\hat{D}^{-1} \hat{\Sigma} \hat{D}^{-1}$.

\section{附录 II: $\mathcal{F}(t, y)$ 的弱收敛}

根据大数定理可知, $\tilde{X}(t, y), B_{x}(t, y)$ 和 $B_{z}(t, y)$ 的极限存在, 分别由 $\tilde{x}(t, y), b_{x}(t, y)$ 和 $b_{z}(t, y)$ 表 
示. 类似于附录 I 中的证明, 我们有

$$
\begin{aligned}
\mathcal{F}(t, y)= & n^{-1 / 2} \sum_{i=1}^{n} \int_{0}^{t}\left\{I\left(X_{i}(u) \leqslant y\right)-\tilde{x}(u, y)\right\} d M_{i}(u) \\
& +2 n^{-1 / 2} \sum_{i=1}^{n} \int_{0}^{t}\left[\int\{I(x(u) \leqslant y)-\tilde{x}(u, y)\} h(x(u)) I(c \geqslant u)\right. \\
& \left.\times \frac{m(m-1) \exp \left\{\beta_{0}^{\prime} x(u)\right\}}{\Lambda_{0}(c)^{2} \exp \left(2 \gamma_{0}^{\prime} z\right)}\left\{\xi_{i}\left(c ; \alpha_{0}\right)+z^{\prime} \eta_{i}\left(\alpha_{0}\right)\right\} d P_{2}(z, c, m, x(u))\right] d \mathcal{A}(u) \\
& -b_{x}(t, y) n^{1 / 2}\left(\hat{\beta}-\beta_{0}\right)-b_{z}(t, y) n^{1 / 2}\left(\hat{\gamma}-\gamma_{0}\right)+o_{p}(1),
\end{aligned}
$$

其中 $d M_{i}(t)=\rho_{i}\left(t ; \gamma_{0}, h\right)^{-1}\left[\Delta_{i}(t) Y_{i}(t) d N_{i}(t)-\Delta_{i}(t) \tilde{\Omega}_{i} \exp \left\{\beta_{0}^{\prime} X_{i}(t)+\gamma_{0}^{\prime} Z_{i}\right\} d \mathcal{A}(t)\right]$. 由文献 [23] 中附录 A.5 的证明可知, (A.8) 式右边的前两项是胎紧的. 另外, 由于 $n^{1 / 2}\left(\hat{\beta}-\beta_{0}\right)$ 和 $n^{1 / 2}\left(\hat{\gamma}-\gamma_{0}\right)$ 依分布收 玫, 且 $b_{x}(t, y)$ 和 $b_{z}(t, y)$ 为确定性函数, 因此后两项也是胎紧的, 从而 $\mathcal{F}(t, y)$ 是胎紧的.

由 (A.2), (A.7) 和 (A.8) 知, $\mathcal{F}(t, y)$ 进一步可以写为

$$
\mathcal{F}(t, y)=n^{-1 / 2} \sum_{i=1}^{n} \Upsilon_{i}(t, y)+o_{p}(1)
$$

其中

$$
\begin{aligned}
\Upsilon_{i}(t, y)= & \int_{0}^{t}\left\{I\left(X_{i}(u) \leqslant y\right)-\tilde{x}(u, y)\right\} d M_{i}(u)+2 \int_{0}^{t}\left[\int\{I(x(u) \leqslant y)-\tilde{x}(u, y)\} h(x(u))\right. \\
& \left.\times I(c \geqslant u) \frac{m(m-1) \exp \left\{\beta_{0}^{\prime} x(u)\right\}}{\Lambda_{0}(c)^{2} \exp \left(2 \gamma_{0}^{\prime} z\right)}\left\{\xi_{i}\left(c ; \alpha_{0}\right)+z^{\prime} \eta_{i}\left(\alpha_{0}\right)\right\} d P_{2}(z, c, m, x(u))\right] d \mathcal{A}(u) \\
& -b_{x}(t, y) D^{-1} \Psi_{i}-b_{z}(t, y) \eta_{i}\left(\alpha_{0}\right) .
\end{aligned}
$$

由多元中心极限定理, 并结合 $\mathcal{F}(t, y)$ 的胎紧性, 可知 $\mathcal{F}(t, y)$ 弱收玫到一个均值为零的 Gauss 过程, 其 在 $(t, y)$ 和 $\left(t^{\dagger}, y^{\dagger}\right)$ 处的协方差函数为 $E\left\{\Upsilon_{i}(t, y) \Upsilon_{i}\left(t^{\dagger}, y^{\dagger}\right)\right\}$, 其相合估计由 (8) 式给出.

\section{Analysis of panel data with informative observation and censoring times under biased sampling}

LIU HuanBin, MIAO Rui \& SUN LiuQuan

Abstract Panel data frequently occur in many studies such as longitudinal follow-up studies. In many situations, longitudinal responses may be correlated with observation times as well as censoring time. This paper considers the regression analysis of panel data where these correlations may exist under biased sampling, and a joint modeling approach that uses some latent variables to characterize the correlations is proposed. For inference about regression parameters, estimating equation approaches are developed and asymptotic properties of the proposed estimators are established. The finite sample behavior of the methods is examined through simulation studies, and an application to a data set from a bladder cancer study is provided for illustration.

Keywords: biased sampling, estimating equations, informative observation process, joint modeling, latent variables, panel data

MSC(2000): 62G05, 62N02

doi: $\quad 10.1360 / 012009-1033$ 